\title{
All Subgraphs of a Wheel are 5-Coupled-Choosable*
}

\author{
Sam Barr and Therese Biedl \\ David R. Cheriton School of Computer Science, University of Waterloo, Waterloo, \\ Canada. $\{$ s4barr, biedl $\}$ @uwaterloo.ca
}

\begin{abstract}
A wheel graph consists of a cycle along with a center vertex connected to every vertex in the cycle. In this paper we show that every subgraph of a wheel graph has list coupled chromatic number at most 5, and this coloring can be found in linear time. We further show that ' 5 ' is tight for every wheel graph with at least 5 vertices, and briefly discuss possible generalizations to planar graphs of treewidth 3 .
\end{abstract}

\section{Introduction}

In this paper we study the problem of coupled choosability, the problem of finding a valid coloring given list assignments to every vertex and face of a planar graph. The problem is of great relevance to list coloring 1-planar graphs, as list coupled coloring a planar graph corresponds to list coloring an optimal 1-planar graph. (Detailed definitions will be given in Section 2.) Wang and Lih 13 show that every planar graph is 7-coupled-choosable, and hence every optimal 1-planar graph is 7-choosable. They further show that maximal planar graphs are 6coupled-choosable, planar graphs of maximum degree 3 are 6-coupled-choosable, and outerplanar graphs (and more generally, all $K_{4}$-minor free graphs) are 5coupled-choosable.

The result by Wang and Lih settles the coupled choosability for planar partial 2-trees (which are the same as $K_{4}$-minor free graphs). Initially wishing to investigate the coupled choosability of planar partial 3-trees, in this paper we investigate the coupled choosability of wheel graphs and their subgraphs. In Theorem 2, we show that any subgraph of a wheel is 5-coupled-choosable, and the coloring can be found in linear time. (Prior papers such as [13] did not address the run-time of finding their colorings; it can clearly be done in polynomial time by following the steps of their proof but linear time is not obvious.) In Theorem 3 , we characterize the coupled choosability of wheel graphs by showing that 5 is tight for wheel graphs with at least 5 vertices. In the last section of the paper, we touch upon how these results could be relevant in finding the coupled choosability of planar partial 3-trees.

As for related results, the (non-coupled) choosability of wheel graphs was characterized in a different paper by Wang and Lih [12]: wheels of even order

\footnotetext{
${ }^{*}$ Research of TB supported by NSERC.
} 
have list chromatic number 4, while wheels of odd order have list chromatic number 3. This stands in contrast to our result, as the parity of the number of vertices in the graph does not affect the coupled choosability of wheel graphs. Wang and Lih also show that Halin graphs that are not wheels have list chromatic number 3, while in Theorem 4 we prove the existence of a Halin graph that is not 5-coupled-choosable (in fact, it is not 5 -coupled-colorable).

Our paper is structured as follows: In Section 2 we will go over the necessary definitions and terminology for graphs and graph coloring. In Section 3 we investigate the coupled choosability of wheel graphs. In Section 4 we examine how coupled choosability behaves under certain graph operations. In Section 5 we extend our analysis of wheel graphs to subgraphs of wheels, along with lowerbounding the coupled choosability of wheel graphs. In Section 6 we go over several possible extensions to our results, in particular some conjectures about the coupled-choosability of planar partial 3-trees.

\section{Definitions}

We assume basic familiarity with graph theory (see [3]). In this paper all graphs are finite and connected.

The complete graph $K_{4}$ consists of four vertices and all possible edges between them. A subdivision of a graph $G$ is formed by repeatedly taking some edge $u v \in E(G)$, removing $e$ from $G$, adding a new vertex $x$, and adding edges $u x$ and $x v$. A graph is called $K_{4}$-minor free if none of its subgraphs is a subdivision of $K_{4}$.

We recall that a graph $G$ is called planar if it can be drawn in the plane without edges crossing, and plane if a specific planar drawing $\Gamma$ is given. The maximal regions of $\mathbb{R} \backslash \Gamma$ are called faces; the unbounded region is known as the outer face and all other faces are inner faces. An outerplanar graph is a graph that can be drawn in the plane such that every vertex is on the outer face; such a graph is $K_{4}$-minor free. A bigon is a face that is bounded by two duplicate edges between a pair of vertices. For a plane graph $G$, we use $V(G), E(G)$, and $F(G)$ to denote the set of vertices, the set of edges, and the set of faces of $G$, respectively. The dual graph $G^{*}$ of a plane graph is obtained by exchanging the roles of vertices and faces, i.e., $G^{*}$ has a vertex for every face of $G$, and an edge $\left(f_{1}, f_{2}\right)$ for every common edge of the two corresponding faces $f_{1}, f_{2}$ in $G$.

A list assignment is a map $L$ that assigns a set of colors for each vertex or face in $V(G) \cup F(G)$. A coupled coloring with respect to $L$ is a map $c$ such that $c(x) \in L(x)$ for every $x \in V(G) \cup F(G)$, and $L(x) \neq L(y)$ for incident or adjacent elements $x, y \in V(G) \cup F(G)$. If such a map $c$ exists, then we say that $G$ is $L$ coupled-choosable. If $G$ is $L$-coupled-choosable for every $L$ such that $|L(x)|=k$ for every $x \in V(G) \cup F(G)$, then we say that $G$ is $k$-coupled-choosable. The smallest integer $k$ such that such that $G$ is $k$-coupled-choosable is called the list coupled chromatic number of $G$ and denoted $\chi_{v f}^{L}(G)$. Observe that a list coupled coloring of a graph $G$ implies a list coupled coloring of the dual graph $G^{*}$, since 
the roles of the vertices and the faces is exchanged but incidences/adjacencies stay the same. Hence, we have $\chi_{v f}^{L}(G)=\chi_{v f}^{L}\left(G^{*}\right)$.

A natural way to express the list coupled chromatic number is to define a new graph $X(G)$ with vertices for all vertices and faces of $G$ and edges whenever the vertices and faces $G$ are adjacent/incident. This graph $X(G)$ is 1-planar, i.e., can be drawn in the plane with at most one crossing per edge. In fact, if $G$ is 3-connected then $X(G)$ is an optimal 1-planar graph, i.e., it is simple and has the maximum-possible $4 n-8$ edges. (All optimal 1-planar graphs can be obtained in this fashion [9].) A coupled coloring of $G$ corresponds to a vertex coloring of $X(G)$, i.e., a coloring of the vertices such that adjacent vertices have different colors. When restricting a vertex coloring to given lists $L$, then the respective terms are $L$-choosable, $k$-choosable, and the list chromatic number $\chi^{L}(X)$.

The wheel graph $W_{n}$ is formed by starting with a cycle $C_{n-1}$ on $n-1$ vertices (the outer cycle), adding a center vertex inside the cycle and adding a spokeedge from the center vertex to every vertex on the cycle. We will label the center vertex and the outer face of the wheel graph as $x_{0}$ and $f_{0}$, respectively. We further label the vertices in the outer cycle as $x_{1}, \ldots, x_{n-1}$, and label the inner faces as $f_{1}, \ldots, f_{n-1}$ such that $x_{i}$ is incident to $f_{i}$ and $f_{i+1}$ for $1 \leq i<n-1$, and $x_{n-1}$ is adjacent to $f_{n-1}$ and $f_{1}$ (see Figure 1 ).

\section{Coupled Choosability of Wheel Graphs}

In order to prove the desired result for all subgraphs of the wheel graph, we first determine the coupled choosability of the wheel graph itself. It will be helpful to recall the following result relating the choosability of a graph to the maximum degree; it is an analogue to Brook's theorem and similarly upper-bounds the chromatic number of a graph by its maximum degree.

Lemma 1. (Erdös, Rubin, and Taylor [5]) Let $G$ be a connected graph that is neither an odd cycle nor a complete graph. Then $G$ is $\Delta(G)$-choosable.

Our main result in this section is:

Lemma 2. Every wheel graph $W_{n}, n \geq 4$, is 5-coupled-choosable.

Proof. For $n=4, W_{4}$ is the complete graph $K_{4}$. Wang and Lih [13] proved that $\chi_{v f}^{L}\left(K_{4}\right)=4$, so we assume $n \geq 5$. Let $L$ be a color assignment for $W_{n}$ such that $|L(y)|=5$ for every $y \in V\left(W_{n}\right) \cup F\left(W_{n}\right)$. Our goal is to find a coupled coloring with respect to $L$. Since $x_{0}$ and $f_{0}$ are both adjacent to all remaining vertices, we will color them first and then color the rest of $X\left(W_{n}\right)$. We will use $X_{n}$ as a shortcut for $X\left(W_{n}\right) \backslash\left\{x_{0}, f_{0}\right\}$. Observe that $\left|V\left(X_{n}\right)\right|=2 n-2$ and that $X_{n}$ is 4-regular (see Figure 1). Furthermore, it suffices to find a vertex-colouring of $X_{n}$ with respect to $L$, plus two suitable colors in $L\left(x_{0}\right)$ and $L\left(f_{0}\right)$ for $x_{0}$ and $f_{0}$. We have two cases:

Case 1: $L\left(x_{0}\right) \cap L\left(f_{0}\right) \neq \emptyset$. Let $a \in L\left(x_{0}\right) \cap L\left(f_{0}\right)$, and assign color $a$ to $x_{0}$ and $f_{0}$. Observe that $|L(y) \backslash\{a\}| \geq 4$ for every $y \in V\left(X_{n}\right)$ and $X_{n}$ has maximum 

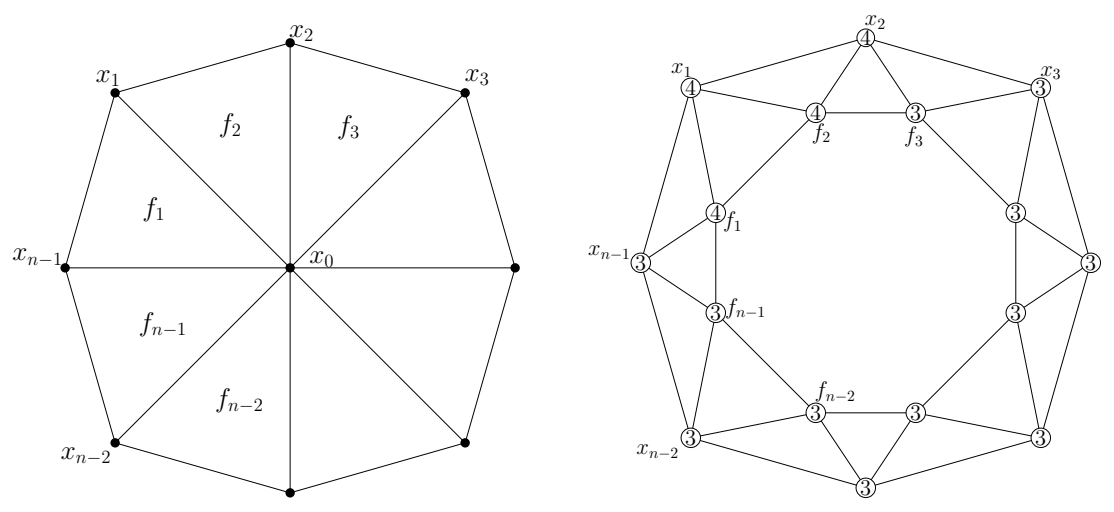

Fig. 1. The graph $W_{9}$ (left) and $X_{9}$ (right). Circled numbers indicate a lower bound on the list-length in $L^{\prime}$.

degree 4. Moreover $\left|X_{n}\right|=2 n-2$ is even, so $X_{n}$ is not an odd cycle. Also $x_{1}$ and $x_{3}$ are not adjacent by $n \geq 5$, so $X_{n}$ is not a complete graph. Therefore, by Lemma 1, we have a list coloring of the vertices of $X_{n}$ that only uses colors in $L \backslash\{a\}$, which in turn implies an $L$-list-coloring of the vertices and faces of $W_{n}$.

Case 2: $L\left(x_{0}\right) \cap L\left(f_{0}\right)=\emptyset$. We find suitable colors for $x_{0}$ and $f_{0}$ by imitating the method used for $K_{4}$ in [13] (but adapted here to 5 colors). Define color-pairs $S:=\left\{\{a, b\}: a \in L\left(x_{0}\right), b \in L\left(f_{0}\right)\right\}$. By case-assumption $|S|=25$.

We claim that $|\{s \in S: s \subseteq L(y)\}| \leq 6$ for any $y \in V\left(X_{n}\right)$. To see this, let $y \in V\left(X_{n}\right)$, and consider the disjoint sub-lists $L_{1}:=L(y) \cap L\left(x_{0}\right)$ and $L_{2}:=L(y) \cap L\left(f_{0}\right)$. Since $\left|L_{1}\right|+\left|L_{2}\right| \leq|L(y)|=5$, and $\left|L_{1}\right|$ and $\left|L_{2}\right|$ are integers, we have

$$
|\{s \in S: s \subseteq L(y)\}|=\left|L_{1} \times L_{2}\right|=\left|L_{1}\right| \cdot\left|L_{2}\right| \leq 6 .
$$

Therefore, color-pairs of $S$ appear as subsets of lists in $X_{n}$ at most

$$
\sum_{y \in X_{n}}|\{s \in S: s \subseteq L(y)\}| \leq(2 n-2) \cdot 6=12 n-12
$$

times. By $|S|=25$, some element $\left\{a^{\prime}, b^{\prime}\right\}$ of $S$ appears at most

$$
\frac{12 n-12}{25}<\frac{n-1}{2}
$$

times as a subset of a list in $X_{n}$. Color $x_{0}$ with $a^{\prime}$ and $f_{0}$ with $b^{\prime}$. For $y \in V\left(X_{n}\right)$, define $L^{\prime}(y):=L(y) \backslash\left\{a^{\prime}, b^{\prime}\right\}$. For any $y \in V\left(X_{n}\right)$, we have $3 \leq\left|L^{\prime}(y)\right| \leq 5$. We call $y$ a 3-vertex if $\left|L^{\prime}(y)\right|=3$ (this implies $\left\{a^{\prime}, b^{\prime}\right\} \subset L(y)$ ), and a 4-vertex otherwise. From our choice of colors $a^{\prime}$ and $b^{\prime}$, we have

$$
\frac{\mid\left\{y \in V\left(X_{n}\right): y \text { is a } 3 \text {-vertex }\right\} \mid}{\left|V\left(X_{n}\right)\right|}<\frac{(n-1) / 2}{2 n-2}=\frac{1}{4}
$$


Therefore, more than three quarters of the vertices of $X_{n}$ are 4 -vertices. Consider the cyclic enumeration

$$
\sigma:=\left\langle f_{1}, x_{1}, f_{2}, x_{2}, \ldots, f_{n-1}, x_{n-1}\right\rangle
$$

of the vertices of $X_{n}$. Since strictly more than $\frac{3}{4}\left|V\left(X_{n}\right)\right|$ of the vertices are 4 -vertices, we have four consecutive 4-vertices in $\sigma$. Up to exchange of $f_{i}$ and $x_{i}$ and renumbering, we may assume that $f_{1}, x_{1}, f_{2}$, and $x_{2}$ are 4 -vertices. Figure 11(right) illustrates the lower bounds on the size of $L^{\prime}$.

We next color $f_{n-1}, x_{n-1}$ and $x_{1}$ and have two sub-cases. If $L^{\prime}\left(f_{n-1}\right) \cap$ $L^{\prime}\left(x_{1}\right) \neq \emptyset$, then color $f_{n-1}$ and $x_{1}$ with the same color. Otherwise, since $\left|L^{\prime}\left(f_{n-1}\right) \cup L^{\prime}\left(x_{1}\right)\right| \geq 7>\left|L\left(f_{1}\right)\right|$, there are colors $p$ and $q$ for $f_{n-1}$ and $x_{1}$ respectively such that at least one of them is not in $L\left(f_{1}\right)$, i.e., $\left|L\left(f_{1}\right) \cap\{p, q\}\right| \leq 1$. Pick these colors for $f_{n-1}$ and $x_{1}$. In either case, two vertices adjacent to $x_{n-1}$ have been colored, and $\left|L^{\prime}\left(x_{n-1}\right)\right| \geq 3$, so $x_{n-1}$ will have at least one valid color left, and we pick this color for $x_{n-1}$.

We now have colors $p, q$, and $r$ for $f_{n-1}, x_{1}$, and $x_{n-1}$ (respectively) such that $\left|L^{\prime}\left(f_{1}\right) \cap\{p, q, r\}\right| \leq 2$. Removing these colors from the lists of their neighbors produces new lists $L^{\prime \prime}$ such that

$$
\begin{aligned}
\left|L^{\prime \prime}\left(f_{1}\right)\right| & =\left|L^{\prime}\left(f_{1}\right) \backslash\{p, q, r\}\right| \geq 4-2=2 \\
\left|L^{\prime \prime}\left(f_{2}\right)\right| & =\left|L^{\prime}\left(f_{2}\right) \backslash\{q\}\right| \geq 4-1=3 \\
\left|L^{\prime \prime}\left(x_{2}\right)\right| & =\left|L^{\prime}\left(x_{2}\right) \backslash\{q\}\right| \geq 4-1=3 \\
\left|L^{\prime \prime}\left(x_{n-2}\right)\right| & =\left|L^{\prime}\left(x_{n-2}\right) \backslash\{p, r\}\right| \geq 3-2=1 \\
\left|L^{\prime \prime}\left(f_{n-2}\right)\right| & =\left|L^{\prime}\left(f_{n-2}\right) \backslash\{p\}\right| \geq 3-1=2 \\
\left|L^{\prime \prime}\left(x_{i}\right)\right| & \geq 3 \quad(\text { for all } 3 \leq i \leq n-3) \\
\left|L^{\prime \prime}\left(f_{i}\right)\right| & \geq 3 \quad \text { (for all } 3 \leq i \leq n-3)
\end{aligned}
$$

The figure on the right illustrates these lower bounds on the list-lengths in $L^{\prime \prime}$.

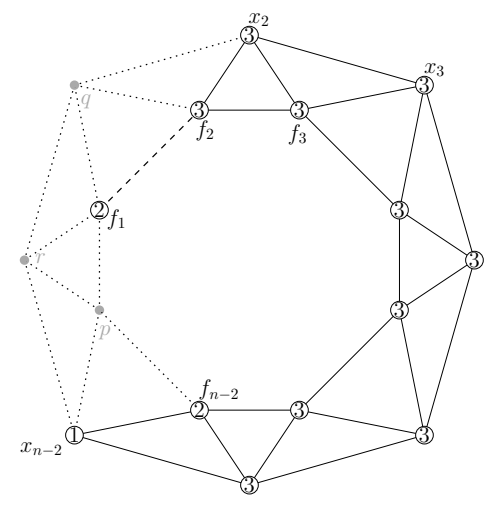

Let $X_{n}^{\prime}:=X_{n} \backslash\left\{f_{n-1}, x_{n-1}, f_{1}, x_{1}\right\}$ ( $X_{n}^{\prime}$ is solid in the above figure) and color it with respect to list assignment $L^{\prime \prime}$. This is feasible since $X_{n}^{\prime}$ is outerplanar and outerplanar graphs are 3 -choosable even if the colors of two consecutive vertices on the outer face are fixed [8] (here we fix the colors for $x_{n-2}$ and $f_{n-2}$ ). This colors all vertices except for $f_{1}$, but $\left|L^{\prime \prime}\left(f_{1}\right)\right| \geq 2$ and $f_{1}$ has only one neighbor in $X_{n}^{\prime}$, so we can give it a color not used by $f_{2}$. Therefore, we have a list vertexcoloring of $X_{n}$ that is compatible with the colors for $x_{0}, f_{0}$ chosen earlier and so implies a list coupled coloring of $W_{n}$.

Note that this coloring can easily be found in linear time. This is obvious in Case 1 due to the linear-time result for Lemma 11 [10. Determining the colors $a, b$ for Case 2 takes linear time since all list-lengths are constant, and then we mostly appeal to list-coloring an outer-planar graph, which can be done in linear time since outer-planar graphs are 2-degenerate. 


\section{Coupled Choosability Under Graph Operations}

In Section 5 we seek to prove that all subgraphs of a wheel are 5-coupledchoosable. In pursuit of this, we examine how various graph operations affect the list-coupled-chromatic number. First, in contrast to list-vertex-coloring, there is no clear relationship between the list coupled chromatic number of a graph and the list coupled chromatic number of its subgraphs. Indeed, a subgraph may have larger list coupled chromatic number.

Observation 1. There exists a plane graph $G$ with subgraph $H \subseteq G$ such that $\chi_{v f}^{L}(H)>\chi_{v f}^{L}(G)$

Proof. Let $H$ be the graph obtained by deleting one edge of $K_{4}$; see Figure 2 . From Theorem 10 of [13, we know that the graph $K_{4}$ is 4-coupled-choosable, i.e., $\chi_{v f}^{L}\left(K_{4}\right)=4$. But in graph $H$, the incidences and adjacencies between $x_{0}, x_{1}, x_{2}, f_{2}$, and $f^{\prime}$ form a $K_{5}$, and therefore $\chi_{v f}^{L}(H) \geq 5>4=\chi_{v f}^{L}\left(K_{4}\right)$.
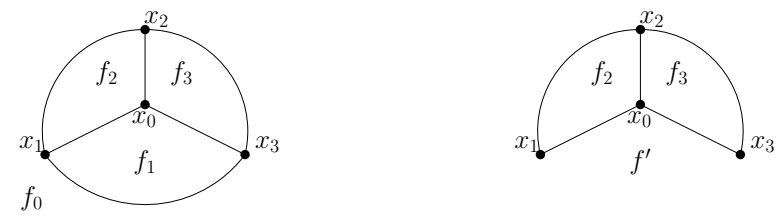

Fig. 2. $K_{4}$ and subgraph $H$. Observe that $\chi_{v f}^{L}(H)=5$ since it is outerplanar.

Other graph operations are better behaved in this respect. For instance, there is a clear relationship between the coupled choosability of some graph $G$ and the coupled choosability of any subdivision of $G$.

Lemma 3. For any plane graph $G$, any subdivision $H$ of $G$ is $\max \left\{5, \chi_{v f}^{L}(G)\right\}$ coupled-choosable.

Proof. Let $L$ be a list assignment for $H$ such that $|L(x)|=\max \left\{5, \chi_{v f}^{L}(G)\right\}$ for every vertex and face of $H$. We prove the statement by induction on the number of subdivisions performed on $G$ to obtain $H$. If $H$ is the result of subdividing the edges of $G$ zero times, then $H=G$ and so trivially any $L$-coupled-coloring of $G$ is an $L$-coupled-coloring of $H$.

Otherwise, $H$ was the result of performing $k+1$ subdivisions on $G$ for some $k \geq 0$. In particular, $H$ is the result of subdividing a single edge of some graph $H^{\prime}$, where $H^{\prime}$ was the result of performing $k$ subdivisions on $G$. Let $u v \in E\left(H^{\prime}\right)$ be the edge of $H^{\prime}$ that was subdivided, and let $x$ be the vertex which was added. By the inductive hypothesis, $H^{\prime}$ is $\max \left\{5, \chi_{v f}^{L}(G)\right\}$-coupled-choosable. Color the faces of $H$ and the vertices $V(H) \backslash\{x\}$ according to how they would be colored in $H^{\prime}$. Then we only need to color the remaining vertex $x$. Note that $x$ has degree two with neighbors $u$ and $v$. Let $f_{1}$ and $f_{2}$ be the two faces adjacent to the edge 
$u v$ in $H^{\prime}$. Then $u, v, f_{1}$, and $f_{2}$ are the only vertices and faces that are adjacent (respectively incident) to $x$. Hence, after coloring the vertices and faces from $H^{\prime}$, $x$ still has at least $|L(x)|-4 \geq 5-4=1$ color left and can be colored.

This implies another result. For a planar graph $G$, subdividing an edge corresponds in the dual graph $G^{*}$ to duplicating edges to form bigons. Since $\chi_{v f}^{L}(G)=\chi_{v f}^{L}\left(G^{*}\right)$ we therefore have:

Corollary 1. Let $G$ be a plane graph, and $H$ the result of duplicating some edges of $G$ to form bigons. Then $H$ is $\max \left\{5, \chi_{v f}^{L}(G)\right\}$-coupled-choosable.

A similar result can also be had for adding a vertex of degree one to a graph.

Lemma 4. Let $G$ be a planar graph, and let $H$ be $G$ plus a new vertex of degree one. Then $H$ is $\max \left\{3, \chi_{v f}^{L}(G)\right\}$-coupled-choosable.

Proof. Let $x$ be the new vertex, and let $L$ be a list assignment for $H$ such that $|L(y)|=\max \left\{3, \chi_{v f}^{L}(G)\right\}$ for every vertex and face of $H$. Color the faces and vertices of $H-x$ according to how they would be colored in $G$. It remains to color $x$. Since $x$ is adjacent to only one vertex and incident to only one face in $H$, after coloring the vertices and face of $H-x, x$ still has at least $|L(x)|-2 \geq 3-2=1$ color left and can be colored.

Note that for all three of the above lemmas, the coloring of $H$ can be found in constant time, given a suitable coloring of $G$.

Wang and Lih [13] proved that all $K_{4}$-minor free graphs are 5-coupledchoosable, but it is not clear whether their proof leads to a linear-time algorithm to find the coloring. With the above two results, such an algorithm is immediate.

Theorem 1. All K4-minor free graphs are 5-coupled-choosable, and the coloring can be found in linear time.

Proof. It is known (see [4]) that every $K_{4}$-minor free graph $G$ can be obtained from some tree $T$ via a series of duplicating edges, subdividing edges, and adding vertices of degree one. Then by Lemmas 3 and 4 . Corollary 1 , and the 3-coupledchoosability of trees, we have that $G$ is 5 -coupled-choosable.

To find the coloring efficiently, first split $G$ into its 2-connected components $C_{1}, \ldots, C_{d}$ [7]. Then run on each component $C_{i}$ the algorithm that recognizes so-called series-parallel graphs in linear time [11. Since 2-connected $K_{4}$-minor free graphs are series-parallel graphs, this algorithm will succeed on each $C_{i}$, and following the trace of its execution one obtains how to construct $C_{i}$ from a single edge via a series of duplicating edges and subdividing edges. Combining this with the tree of 2-connected components shows how to obtain $G$. Since trees are trivially 3-coupled-colorable (choose a color for the unique face, then find a 2 -coloring of the vertices), and each of our expansion steps takes constant time, we can find the coloring of $G$ in linear time. 


\section{Subgraphs of Wheels}

We now turn to graphs that are subgraphs of wheels. As demonstrated in Observation 1. non-trivial work is required to demonstrate that any subgraph of a wheel graph is also 5-coupled-choosable. The result comes quickly from the results proved in the previous section.

Theorem 2. Let $G$ be a subgraph of a wheel graph $W_{n}, n \geq 4$. Then $G$ is 5-coupled-choosable and the coloring can be found in linear time.

Proof. We examine several possibilities of the structure of $G$.

Case 1: $G=W_{n}$. Then by Lemma $2 G$ is 5 -coupled-choosable, and the coloring can be found in linear time.

Case 2: $G$ is the result of deleting at least one edge or vertex of $W_{n}$ that is on the outer face. Then $G$ is outerplanar and therefore $K_{4}$-minor free, and so by Theorem 1 , $G$ is 5 -coupled-choosable and the coloring can be found in linear time.

Case 3: $G$ is the result of removing the center vertex of $W_{n}$. Then $G=C_{n-1}$ is outerplanar and (as in the previous case) 5-coupled choosable.

Case 4: None of the above. Then all vertices of $W_{n}$ belong to $G$, but we deleted some edges which were not on the outer face. So $G$ is the result of deleting some of the spoke-edges incident to the center vertex. If at most two spokes remain, then $G$ has at most 3 faces and therefore is $K_{4}$-minor free, and hence is 5-coupled-choosable by Theorem 1. If at least three spokes remain, then $G$ is a subdivision of some $W_{k}$ for $k \geq 4$. By Lemmas 2 and $3 G$ is 5 -coupledchoosable, and we can find the coloring in linear time since we can detect all subdivision-vertices by scanning for vertices in linear time.

Having established an upper bound on the list coupled chromatic number of wheel graphs in Lemma2 2 one might wonder whether this bound is tight or not. In [13, it is shown that the graph $K_{4}=W_{4}$ is 4-coupled-choosable. In fact, this is the only wheel graph that is 4-coupled-choosable. For all other wheel graphs, the bound of 5 -coupled-choosability is tight.

Theorem 3. $\chi_{v f}^{L}\left(W_{n}\right)=5$, for $n \geq 5$.

Proof. From Lemma 2, we know that all wheel graphs are 5-coupled-choosable. It remains to show that they are not 4-coupled-choosable for $n \geq 5$.

For $n=5,6$, we consider the list assignment $L$ such that $L(y)=\{1,2,3,4\}$ for every $y \in V\left(W_{n}\right) \cup F\left(W_{n}\right)$. (So these graphs are not even 4-coupled-colorable.) Assume for contradiction that we have an $L$-coupled-coloring $c$ of $W_{n}$. If $c\left(x_{0}\right) \neq$ $c\left(f_{0}\right)$, then this leaves two colors for coloring the triangle $x_{1}, f_{1}, f_{2}$ in $X_{n}$, impossible. Hence $c\left(x_{0}\right)=c\left(f_{0}\right)$, say they are both colored 4 . Then we have an $L^{\prime}$-coloring of $X_{n}$ with lists $L^{\prime}(y):=L(y) \backslash\{4\}=\{1,2,3\}$.

Observe that for $X_{5}$ and $X_{6}$, any putative $L^{\prime}$-coloring would be unique up to renaming the colors, since once we have colored one triangle, every other vertex 
can be reached via a sequence of triangles. One verifies that for these graphs (and indeed every $X_{k}$ where $k-1$ is not divisible by 3 ), attempting such a 3 -coloring leads to a contradiction (see Figure 3). This proves Theorem 3 for $n=5,6$.

For $n \geq 7$, we construct a list assignment $L$ such that $W_{n}$ is not $L$-coupledchoosable. Set $L\left(x_{0}\right)=\{1,2,3,4\}$ and $L\left(f_{0}\right)=\{5,6,7,8\}$. We further define:

$$
\begin{aligned}
& L\left(f_{1}\right)=L\left(x_{1}\right)=L\left(f_{2}\right)=\{1,2,5,6\} \\
& L\left(x_{2}\right)=L\left(f_{3}\right)=L\left(x_{3}\right)=\{1,2,7,8\} \\
& L\left(f_{4}\right)=L\left(x_{4}\right)=L\left(f_{5}\right)=\{3,4,5,6\} \\
& L\left(x_{5}\right)=L\left(f_{6}\right)=L\left(x_{6}\right)=\{3,4,7,8\}
\end{aligned}
$$

Observe that each of these triples forms a triangle in $X_{n}$, and for any $a \in$ $\{1,2,3,4\}$ and $b \in\{5,6,7,8\}$, one of these triangles has colors $\{a, b, x, y\}$ for some colors $x, y$. Assume for contradiction that we have an $L$-coupled-coloring $c$ of $W_{n}$. Up to symmetry, assume $c\left(x_{0}\right)=1$ and $c\left(f_{0}\right)=5$. But then $f_{1}, x_{1}$, and $f_{2}$ have two colors left, and therefore cannot be colored, a contradiction.
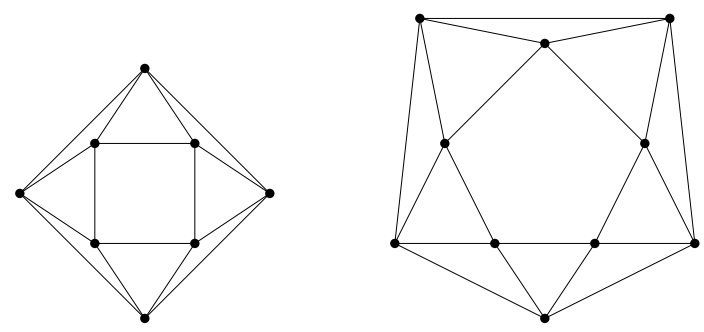

Fig. 3. The graphs $X_{5}$ (left) and $X_{6}$ (right).

With this, we can characterize the coupled choosability of wheel graphs.

Corollary 2. For a wheel graph $W_{n}$, we have $\chi_{v f}^{L}\left(W_{n}\right)=\min \{5, n\}$.

\section{Towards Partial 3-trees}

Our investigation of wheel graphs was motivated by wanting to determine the coupled choosability number of planar partial 3-trees. To define these, we first define Apollonian networks recusively as follows. A triangle is an Apollonian network. If $G$ is an Apollonian network, and $f$ is a face of $G$ (necessarily a triangle) that is not the outer-face, then the graph obtained by stellating face $f$ is also an Apollonian network. Here stellating means the operation of inserting a new vertex $v$ inside face $f$ and making it adjacent to all vertices of $f$. A planar partial 3-tree is a graph that is a subgraph of an Apollonian network 
(see Figure 4). (This definition is different, but equivalent, to the "standard" definition of partial 3-trees via treewidth or via chordal supergraphs with cliquesize 4 [1].) We offer the following conjecture:

Conjecture 1. Every planar partial 3-tree is 6-coupled-choosable.

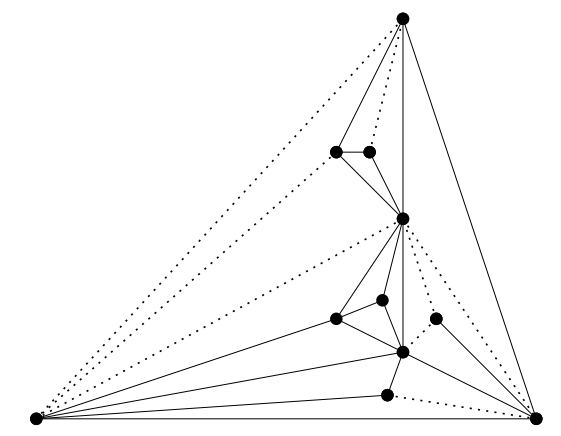

Fig. 4. A planar partial 3-tree. Dotted edges show the Apollonian network.

Note that the conjecture holds for Apollonian networks, since these are maximal planar graphs and these are known to be 6-coupled-choosable [13. But this does not imply 6-coupled-choosability of subgraphs, and so the conjecture remains open.

Towards the conjecture, we studied several graph classes that are planar partial 3-trees (and generalize wheels). One such class of graphs are the Halin graphs, which are defined by starting with a tree $T$ and adding a cycle between the leaves of $T$. See also the solid edges in Figure 5. Wheel graphs are the special case of Halin graphs where $T$ is a star graph. A second class of planar partial 3 -trees are the stellated outer-planar graphs, obtained by starting with some outerplanar graph $G$, and stellating the outer-face. See also the dashed edges in Figure 5. Wheel graphs are the special case of stellated outerplanar graphs where the outerplanar graph is a cycle.

One can easily see that Halin graphs are exactly the duals of stellated outerplanar graphs. Therefore, any list coupled coloring of a stellated outerplanar graph corresponds to a list coupled coloring of a Halin graph. Unfortunately, our upper bound for the coupled choosability of wheel graphs does not in general extend to Halin graphs.

Theorem 4. There exists a stellated outerplanar graph (equivalently a Halin graph) that is not 5-coupled-colorable (in particular therefore it is not 5-coupledchoosable).

Proof. The Halin-graph $G$ is the triangular prism, see Figure 5 where we also show the dual graph $G^{*}$ and the 1-planar graph $X(G)$. The claim holds if we show that there is no 5 -coloring of the vertices of $X(G)$. 
Assume for contradiction that $X(G)$ had a 5-coloring; up to symmetry we may assume that the triangle formed by the three degree-4-faces of $G$ is colored $1,2,3$. Let $\left(t, t^{\prime}\right)$ be the edge that crosses the edge colored with 2 and 3 . Vertices $t, t^{\prime}$ are colored with 1,4 or 5 ; up to renaming of colors 4 and 5 hence one of them is colored 4 .

Starting with this coloring, propagate restrictions on the possible colors to other vertices of $X(G)$ along the numerous copies of $K_{4}$ (note that all vertices other than $t, t^{\prime}$ are adjacent to the one colored 1$)$. This leads to a triangle that has only two possible colors left, a contradiction.

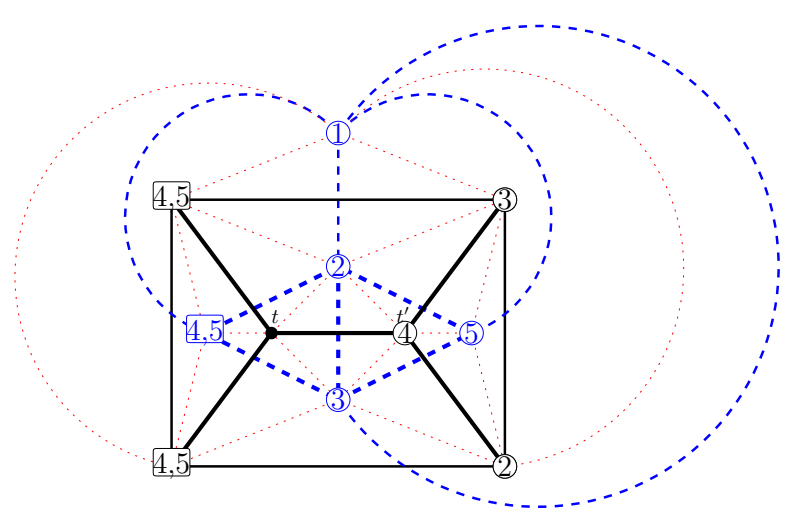

Fig. 5. A Halin-graph $G$ (black solid; the tree is bold), and the dual graph $G^{*}$ (blue dashed) which is a stellated outerplanar graph (the outerplanar graph is bold). Taking both, and adding the face-vertex incidences (red dotted) gives graph $X(G)$. We also show the only possible 5-coloring (up to symmetry) of $X(G)$, which leads to a contradiction since a triangle must be colored with 2 colors.

In particular, this shows that we cannot replace ' 6 ' by ' 5 ' in Conjecture 1 . We also remark that, in line with Observation 1, a supergraph of the triangular prism is 5-coupled-colorable. Namely, one can insert diagonals in the degree-4 faces and obtain the octahedron. An octahedron is 3-colorable because all faces are triangles and the vertex-degrees are even. The dual graph (which is the cube) is bipartite and hence 2-colorable. Therefore, using disjoint sets of colors for the primal and the dual graph, we get a 5-coupled-coloring of the octahedron.

Returning to wheel graphs, Theorem 4 shows that wheels are strictly better (as far as coupled choosability is concerned) than Halin-graphs. Now we study a second graph class that lies between the wheels and the planar partial 3-trees. These are the IO-graphs, which are the planar graphs that can be obtained by adding an independent set to the interior faces of an outerplanar graph (see Figure 60. Certainly any subgraph of a wheel is an IO graph.

Conjecture 2. Every IO-graph is 5-coupled choosable. 
We studied subgraphs of wheel graphs because they may be an important stepping stone towards Conjecture 2 In particular, consider some IO-graph $G$. obtained from an outerplanar graph $O$ and independent set $I$. Let $G^{+}$be a maximal IO-graph containing $G$, i.e., add edges to $G$ for as long as the result is simple and an IO-graph. Then $G^{+}$is a tree of wheels, where each wheel consists of a vertex $x \in I$ with its neighbours, and the wheels have been glued together at edges. Correspondingly $G$ is a tree of subgraphs of wheels. It may be possible to use Theorem 2 (enhanced with further restrictions on the coloring of some parts) to prove Conjecture 2 by building a coloring of $G$ incrementally in this tree, but this remains future work.

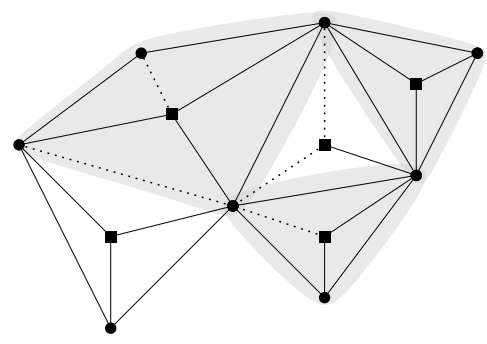

Fig. 6. An IO graph $G$ consists of an outerplanar graph (circles) and an independent set (squares). Dotted edges at added to obtain $G^{+}$, and some of the wheels used to build $G^{+}$are shaded.

We end with some other open questions surrounding list-colorability and listcoupled-colorability. Foremost, is every 1-planar graph 7-list-colorable? Borodin states this to be true [2], but quotes the paper by Wang and Lih [13] which only deals with 7-coupled-choosability. Hence all optimal 1-planar graphs are 7-listcolorable but to our knowledge the problem remains open for 1-planar graphs that are not subgraphs of optimal 1-planar graphs (e.g. any 1-planar graph that contains $K_{6}$ as a subgraph). Second, how easy is it to test whether a planar graph is $k$-coupled-choosable? It is known that testing choosability is $W$ [1]-hard with respect to treewidth, but linear-time solvable for constant treewidth [6]. Do these results transfer to coupled-choosability?

\section{References}

1. Biedl, T., Velázquez, L.R.: Drawing planar 3-trees with given face areas. Computational Geometry: Theory and Applications 46(3), 276-285 (2013). https://doi.org/10.1016/j.comgeo.2012.09.004

2. Borodin, O.V.: Colorings of plane graphs: A survey. Discret. Math. 313(4), 517-539 (2013). https://doi.org/10.1016/j.disc.2012.11.011, https://doi.org/10.1016/j . disc.2012.11.011

3. Diestel, R.: Graph theory, Graduate Texts in Mathematics, vol. 173. Springer, Berlin, fifth edn. (2018). https://doi.org/10.1007/978-3-662-53622-3 
4. Doczkal, C., Pous, D.: Treewidth-Two Graphs as a Free Algebra. In: Potapov, I., Spirakis, P., Worrell, J. (eds.) 43rd International Symposium on Mathematical Foundations of Computer Science (MFCS 2018). Leibniz International Proceedings in Informatics (LIPIcs), vol. 117, pp. 60:160:15. Schloss Dagstuhl-Leibniz-Zentrum fuer Informatik, Dagstuhl, Germany (2018). https://doi.org/10.4230/LIPIcs.MFCS.2018.60 http://drops .dagstuhl. de/opus/volltexte/2018/9642

5. Erdős, P., Rubin, A.L., Taylor, H.: Choosability in graphs. In: Proceedings of the West Coast Conference on Combinatorics, Graph Theory and Computing (Humboldt State Univ., Arcata, Calif., 1979). pp. 125-157. Congress. Numer., XXVI, Utilitas Math., Winnipeg, Man. (1980)

6. Fellows, M.R., Fomin, F.V., Lokshtanov, D., Rosamond, F.A., Saurabh, S., Szeider, S., Thomassen, C.: On the complexity of some colorful problems parameterized by treewidth. Inf. Comput. 209(2), 143-153 (2011). https://doi.org/10.1016/j.ic.2010.11.026, https://doi.org/10.1016/j.ic.2010. 11.026

7. Hopcroft, J.E., Tarjan, R.E.: Efficient algorithms for graph manipulation. Communications of the ACM 16(6) (June 1973)

8. Hutchinson, J.P.: On list-coloring extendable outerplanar graphs. Ars Math. Contemp. 5(1), 171-184 (2012). https://doi.org/10.26493/1855-3974.179.189

9. Schumacher, H.: Zur Struktur 1-planarer Graphen. Mathematische Nachrichten 125, 291-300 (1986)

10. Skulrattanakulchai, S.: $\Delta$-list vertex coloring in linear time. Information Processing Letters 98(3), 101 - 106 (2006). https://doi.org/10.1016/j.ipl.2005.12.007

11. Valdes, J., Tarjan, R.E., Lawler, E.L.: The recognition of series parallel digraphs. SIAM J. Comput. 11(2), 298-313 (1982). https://doi.org/10.1137/0211023, https: //doi.org/10.1137/0211023

12. Wang, W., Lih, K.W.: List coloring Halin graphs. Ars Combin. 77, 53-63 (2005)

13. Wang, W., Lih, K.W.: Coupled choosability of plane graphs. J. Graph Theory 58(1), 27-44 (2008). https://doi.org/10.1002/jgt.20292 